2016-04-19

\title{
Theoretical Modeling of the Effect of Polymer Chain Immobilization Rates on Holographic Recording in Photopolymers
}

\author{
Dana Mackey \\ Technological University Dublin, dana.mackey@tudublin.ie \\ Paul O'Reilly \\ Technological University Dublin, paul.oreilly@tudublin.ie \\ Izabela Naydenova \\ Technological University Dublin, izabela.naydenova@tudublin.ie
}

Follow this and additional works at: https://arrow.tudublin.ie/scschmatart

Part of the Optics Commons, and the Partial Differential Equations Commons

\section{Recommended Citation \\ Mackey, D., O'Reilly, P. \& Naydenova, I. (2016). Theoretical modeling of the effect of polymer chain immobilization rates on holographic recording in photopolymers. Journal of the Optical Society of America A, 33(5), pp.920-929. http://dx.doi.org/10.1364/JOSAA.33.000920}

This Article is brought to you for free and open access by the School of Mathematics at ARROW@TU Dublin. It has been accepted for inclusion in Articles by an authorized administrator of ARROW@TU Dublin. For more information, please contact arrow.admin@tudublin.ie, aisling.coyne@tudublin.ie,gerard.connolly@tudublin.ie.

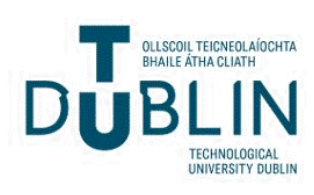




\title{
Theoretical modeling of the effect of polymer chain immobilization rates on holographic recording in photopolymers
}

\author{
Dana Mackey ${ }^{1} \quad$ Paul O'Reilly ${ }^{1} \quad$ Izabela Naydenova ${ }^{2}$
}

${ }^{1}$ School of Mathematical Sciences, Dublin Institute of Technology, Kevin Street, Dublin 8, Ireland

${ }^{2}$ Centre for Industrial and Engineering Optics, Dublin Institute of Technology, Kevin Street, Dublin 8, Ireland

(Corresponding author: dana.mackey@dit.ie)

\begin{abstract}
This paper introduces an improved mathematical model for holographic grating formation in an acrylamide-based photopolymer, which consists of partial differential equations derived from physical laws. The model is based on the two-way diffusion theory of [12], which assumes short polymer chains are free to diffuse, and generalizes a similar model presented in [18] by introducing an immobilization rate governed by chain growth and cross-linking. Numerical simulations were carried out in order to investigate the behaviour of the photopolymer system for short and long exposures, with particular emphasis on the effect of recording parameters (such as illumination frequency and intensity), as well as material permeability, on refractive index modulation, refractive index profile and grating distortion. The model reproduces many well-known experimental observations, such as decrease of refractive index modulation at high spatial frequencies and appearance of higher harmonics in the refractive index profile when the diffusion rate is much slower than polymerization rate. These properties are supported by a theoretical investigation which uses perturbation techniques to approximate the solution over various time scales.
\end{abstract}

\section{Introduction}

Holography has many applications such as holographic displays, optical elements and sensors, security holograms and holographic data storage. A 
hologram is essentially a recording of an interference pattern created by an object beam and a reference beam in a photosensitive material; in all applications the accuracy with which this pattern is copied and the grating strength are crucial for the performance of the hologram. Photopolymer systems (which usually consist of one or two monomers, photoinitiator and sensitizing dye, all dispersed in a binder matrix) are often the material of choice in holographic patterning because of qualities such as versatility, selfprocessing nature, good dynamic range and relatively low cost.

Holographic recording in photopolymer systems has been studied theoretically and experimentally by several authors, [1]-[13]. Early diffusion models describing holographic recording in photopolymers based on free radical polymerization predict that the key factor that controls dynamics and final properties of the recorded holographic grating (refractive index spatial profile and modulation) is determined by the ratio between the rate of polymerization and the monomer diffusion rate. Experimental evaluation of these two rates is important for determination of the optimal conditions for holographic recording in a particular photopolymer system, but this is not always straightforward since in reality both rates are time dependent. The diffusion rate is determined by the matrix permeability and the size of the monomer molecules, while the polymerization rate can be controlled by the recording intensity and also depends on the reactivity of the monomer and efficiency of the initiator. Two extreme regimes of holographic recording at given spatial frequency were distinguished with respect to the ratio of the characteristic diffusion and polymerization rates, [1], [12], [14]. At recording conditions for which the diffusion rate is faster than the polymerization rate the grating profile closely resembles the one of the recording interference pattern. By contrast, when the monomer diffusion rate is slower than the polymerization rate, deviation from the sinusoidal profile of the grating is observed.

A common aim of theoretical models reported in the literature in recent years has been the analysis of the poor response of photopolymers at high spatial frequency, a phenomenon which cannot be described by a simple monomer diffusion model such as [1] (which assumes that growing polymer chains remain at their place of origin). Instead, the low diffraction efficiency at high spatial frequencies has been explained using two approaches, both referring to non-local behaviour (that is, the response of the material at a given point and time depends on what happens at other points and times in the medium). The non-local photopolymerization driven diffusion model (NPDD), [8], [9], [10], [11], [15], assumes that the polymer chains grow away from their initiation point and this is the main factor contribut- 
ing to the "spreading" of the polymer and smearing of the refractive index contrast. This model predicts that improvement at high spatial frequencies can be achieved if, during the holographic recording, shorter polymer chains are created. It has been suggested that the incorporation of a chain transfer agent in the photopolymer composition will improve the diffraction efficiency at high spatial frequency. The reported experimental results from recording in materials containing chain transfer agents demonstrate only marginal improvement of the response at spatial frequency higher than 3000 lines/mm, [16]. An alternative approach to the NPDD theory is to take into account the diffusion of short polymer chains (such as oligomers) away from the bright fringes, which could be responsible for reducing the refractive index modulation and the decrease of diffraction efficiency at high spatial frequencies. This approach is exemplified by the two-way diffusion model, [12], [17], [18], and the immobilization-diffusion model, [19]. Both models account for monomer and polymer diffusion and distinguish between short polymer chains capable of diffusing and long polymer chains that are immobile. In the latter model there is a gradual decrease of polymer mobility depending on length, while in the former we make a sharp distinction between mobile and immobile species.

It has been demonstrated that these models can satisfactorily predict the poor high spatial frequency response in highly permeable photopolymers. The two-way diffusion model also predicts that the improvement of the high spatial frequency response of such systems must be directed towards decreasing the permeability of the photopolymer matrix and avoiding the creation of diffusing short polymer chains. The model has enabled the optimization of the high spatial frequency response of photopolymers, to the extent that full colour reflection holography, [20], is now well established, along with a range of colour changing holographic sensors, [21], [22]. This was achieved by the use of a polymer binder with a lower permeability. It is likely that both diffusion of mobile polymer chains and non-local polymerization processes contribute to the limited spatial resolution in photopolymers, the dominating process depending on the permeability of the recording material and the exposure conditions.

Another model considering the role of the diffusion of mobile polymer chains has been reported recently, [23]. This model is used to analyse the reaction-diffusion kinetics of two-chemistry diffusive photopolymers. It offers a general strategy for characterizing the reaction-diffusion kinetics of photopolymer media in which key processes are decoupled and independently measured.

To support the two-way diffusion theory, a new mathematical model for 
the formation of a weak grating after short exposure time was proposed, [18], [24], which consists of partial differential equations describing monomer and short polymer diffusion, together with photopolymerization and growth of polymer chains until an immobile state is reached. The impact of diffusion coefficients, polymerization rate, intensity and spatial frequency of recording on the properties of weak diffraction gratings were investigated by numerical simulations which successfully reproduced the experimental observations of poor high spatial frequency response.

In this paper, an improved version of this mathematical model is presented, proposing a new mechanism for immobilization (the dynamical process by which diffusing polymers are converted into immobile chains). It is suggested that the immobilization is due to chemical reaction of two polymer chains creating a larger polymer chain or cross-linking of two polymer chains. Moreover, we now focus on the long term behaviour of the equations, which gives insight into the evolution of the monomer and polymer concentrations and refractive index for longer exposure times (comparable with times used in typical holographic recordings). Section 3 presents numerical simulations showing the dependence of the refractive index spatial profile and refractive index modulation on recording frequency and matrix permeability (reflected in the mathematical model by the immobilization rate) for low, moderate and high intensity recording. A study of the dependence on intensity of recording was carried out since the results can be used as a practical guide in choosing the optimal experimental conditions. Finally, Section 4 presents a theoretical analysis of the two extreme regimes mentioned above (monomer diffusion rate much slower or much larger than polymerization rate), with special emphasis on studying the differences in grating strength and deviations of the refractive index spatial profile from the initial sinusoidal illumination pattern.

The results demonstrate that it is possible to simulate the poor spatial frequency response of photopolymers by taking into account the mobile chains diffusion. The simulations can also be used to predict optimal conditions of recording at given spatial frequency in order to achieve maximum refractive index modulation and diffraction efficiency and better fidelity of the recorded interference pattern. 


\section{The mathematical model}

The photopolymer is exposed to two coherent beams of intensities $I_{1}$ and $I_{2}$ which create the following illumination pattern

$$
I(x)=I_{0}(1+V \cos (k x)),
$$

where $k$ is the grating wavenumber, $I_{0}=I_{1}+I_{2}$ and $V=2 \sqrt{I_{1} I_{2}} /\left(I_{1}+I_{2}\right)$ are the overall intensity and visibility of the interference pattern, respectively. The holographic grating formation then proceeds in three steps: initiation, propagation and termination. Upon illumination, the sensitizing dye absorbs a photon and reacts with the electron donor to produce free radicals; in the presence of monomer these free radicals initiate polymerization. It is assumed that direct absorption of monomer and polymer at this recording beam wavelength is negligible. During the propagation step, free radicals and monomer molecules interact and produce growing polymer chains. At the termination step, two free radicals or two polymer chains interact and the polymer chains stop growing. Following exposure to such an interference pattern, the faster consumption of monomer in the high intensity regions sets up a concentration gradient so the free monomer diffuses from dark to bright fringes; in addition we assume that short-chain polymer molecules can also diffuse during recording. The resulting holographic grating is due to the spatial variation of the refractive index caused by changes in the density of monomer and polymer species.

The dynamics of the photopolymer can be described by standard mass conservation equations, whereby monomer, mobile polymer (typically the shorter chains) and immobile polymer are seen as interacting species in a closed system. The evolution of monomer is given by the one-dimensional diffusion model of [1], which assumes that the polymerization rate (which, in our theory, gives the transition from monomer to short or mobile polymer) is proportional to the monomer density and illumination rate. This model was generalized by adding a diffusion equation for short polymer chains, with a growth term due to polymerization and a decay term which accounts for immobilization, formerly assumed to be proportional to the rate of chain growth, [18]. In what follows, we assume instead that immobilization depends mainly on combination of short chains and on cross-linking and both these processes are now more correctly described as proportional to the square of the mobile polymer density. Finally, the last equation describes the growth of immobile polymer at the immobilization point but this species does not diffuse. 
The processes described above are captured by the following partial differential equations (see also [18], [24])

$$
\begin{aligned}
& \frac{\partial m}{\partial t}=D_{m} \frac{\partial^{2} m}{\partial x^{2}}-\Phi(t) F(x) m \\
& \frac{\partial p}{\partial t}=D_{p} \frac{\partial^{2} p}{\partial x^{2}}+\Phi(t)\left[F(x) m-\Gamma p^{2}\right] \\
& \frac{\partial q}{\partial t}=\Phi(t) \Gamma p^{2}
\end{aligned}
$$

where $m(x, t), p(x, t), q(x, t)$ are the concentrations of monomer, short and long polymers, respectively. Due to the periodic nature of this system we choose to study the equations on a spatial domain given by one grating period, $0 \leq x \leq \Lambda$, where $\Lambda=2 \pi / k$, and $t \geq 0$. If the intensity of the incident beams is weak enough not to produce a nonlinear reaction, the polymerization rate can be assumed proportional to the light intensity and written as $F(x)=F_{0}(1+V \cos (k x))$. Finally, $\Gamma$ is the immobilization rate constant which reflects physical properties of the photopolymer (such as permeability and cross-linking activity) and $\Phi(t)$ is a step function equal to 1 during the light exposure and 0 afterwards.

The initial conditions are

$$
m(x, 0)=m_{0}, p(x, 0)=q(x, 0)=0, \quad 0 \leq x \leq \Lambda,
$$

where $m_{0}$ is the monomer density before photopolymerization, and we impose zero-flux boundary conditions since we expect the resulting concentrations to display the same pattern of minima and maxima as the illumination function:

$$
\frac{\partial m}{\partial x}(x, t)=\frac{\partial p}{\partial x}(x, t)=\frac{\partial q}{\partial x}(x, t)=0, \quad x=0, \Lambda ; t \geq 0 .
$$

For simplicity, the monomer and polymer diffusion coefficients, $D_{m}$ and $D_{p}$, are assumed to be constant in space and time. Since, in reality, diffusivity (especially that of the larger polymer molecules) decreases with polymerization, it would seem that our model overestimates the effect of diffusion. However, this effect is compensated in the long term by increased production of immobile chains, as described by (2), so the polymer diffusion slows down significantly, and the simulations of the refractive index behaviour will be shown in later sections to match experimentally observed patterns.

In order to facilitate the theoretical analysis and the numerical simulations, we introduce the following non-dimensional variables

$$
\bar{x}=\frac{x}{\Lambda} ; \quad \bar{t}=\frac{t}{t_{0}} ; \quad \bar{m}=\frac{m}{m_{0}} ; \quad \bar{p}=\frac{p}{m_{0}} ; \quad \bar{q}=\frac{q}{m_{0}}
$$


and the system becomes (after dropping the bars again)

$$
\begin{aligned}
& \frac{\partial m}{\partial t}=\alpha \frac{\partial^{2} m}{\partial x^{2}}-\Phi(t) \beta f(x) m, \\
& \frac{\partial p}{\partial t}=\alpha \varepsilon \frac{\partial^{2} p}{\partial x^{2}}+\Phi(t)\left[\beta f(x) m-\gamma p^{2}\right] \\
& \frac{\partial q}{\partial t}=\Phi(t) \gamma p^{2}
\end{aligned}
$$

where

$$
\begin{gathered}
\alpha=\frac{D_{m} t_{0}}{\Lambda^{2}}, \quad \varepsilon=\frac{D_{p}}{D_{m}}, \quad \beta=t_{0} F_{0}, \quad \gamma=m_{0} t_{0} \Gamma, \\
f(x)=1+\cos (2 \pi x) .
\end{gathered}
$$

We assume that the parameter $\varepsilon=D_{p} / D_{m}$ is small $(\varepsilon \ll 1)$ since the diffusion of short polymers is always much slower than that of monomers. For example, in all subsequent numerical integrations, we take $D_{m}=10^{-12} \mathrm{~m}^{2} / \mathrm{s}$ and $D_{p}=10^{-14} \mathrm{~m}^{2} / \mathrm{s}$ (in accordance with values determined in [12]) which gives $\varepsilon=10^{-2}$. We choose the reference time $t_{0}=1 \mathrm{~s}$ and we also introduce the nondimensional parameter

$$
K=\frac{\alpha}{\beta}=\frac{D_{m}}{F_{0} \Lambda^{2}},
$$

which measures the ratio of the diffusion rate to polymerization rate. (This parameter was also considered in [1], [8], denoted by $R$.) The nondimensional initial conditions are $m(x, 0)=1, p(x, 0)=q(x, 0)=0$, and the zero-flux boundary conditions are applied at $x=0,1$.

The refractive index of a material consisting of a mixture of components can be calculated with the well-known Lorentz-Lorenz equation:

$$
\frac{n^{2}-1}{n^{2}+2}=\sum_{i} \Phi_{i} \frac{n_{i}^{2}-1}{n_{i}^{2}+2}
$$

where $n$ is the effective refractive index of the mixture, $n_{i}$ are the refractive indices of the components (monomer, polymers and binder) and $\Phi_{i}$ are the volume fractions. Since $\Phi_{m}+\Phi_{p}+\Phi_{q}+\Phi_{b}=1$, we can calculate

$$
\Phi_{m}=\frac{m / \rho_{m}}{m / \rho_{m}+p / \rho_{p}+q / \rho_{q}+b / \rho_{b}},
$$

with similar expressions for $\Phi_{p}$ and $\Phi_{q}$, where $\rho_{m}, \rho_{p}, \rho_{q}$ and $\rho_{b}$ are the densities of monomer, polymers and binder, respectively, while $m, p$ and $q$ 
refer to the non-dimensional (scaled) concentrations, as determined by the system (4)-(6), and $b$ is the similarly scaled binder concentration. Assuming that the monomer initially occupies about $17 \%$ of the total volume, we find that $b=0.83 / 0.17=4.9$. The densities and refractive indices for all components are as follows

$$
\begin{gathered}
\rho_{m}=1.15 \mathrm{~g} / \mathrm{cm}^{3} ; \rho_{p}=\rho_{q}=1.3 \mathrm{~g} / \mathrm{cm}^{3} ; \rho_{b}=1.19 \mathrm{~g} / \mathrm{cm}^{3} ; \\
n_{m}=1.49 ; n_{p}=n_{q}=1.52 ; n_{b}=1.48 .
\end{gathered}
$$

These values are consistent with previously published values by other authors modelling acrylamide-based photopolymers (see, for example, [16], [18]). The refractive index calculated by (8) is a function of fringe position and time, $n=n(x, t)$, and has a nonlinear dependence on $m, p$ and $q$, the concentrations of the photopolymer components. When the exposure is stopped early, the monomer and short polymer will quickly diffuse to spatially homogeneous states therefore the refractive index spatial variation will be completely determined by that of immobile polymer.

The refractive index has the Fourier cosine expansion

$$
n(x, t)=n_{0}(t)+n_{1}(t) \cos (2 \pi x)+n_{2}(t) \cos (4 \pi x)+\cdots
$$

and, if we let

$$
\widetilde{n}(x, t)=n_{0}(t)+n_{1}(t) \cos (2 \pi x)
$$

be the sinusoidal approximation, we define the grating distortion as

$$
d^{2}(t)=\int_{0}^{1}[n(x, t)-\widetilde{n}(x, t)]^{2} d x .
$$

Since $d^{2}=n_{2}^{2}+n_{3}^{2}+\cdots$ (by Parseval's identity) it follows that the function $d(t)$ contains information regarding all higher harmonic amplitudes and can therefore be used as a measure of the refractive index departure from the ideal sinusoidal profile.

The absolute value of the first harmonic amplitude, $\left|n_{1}(t)\right|$, is frequently used in the literature as a measure of grating strength and an approximation to the refractive index modulation,

$$
\left|n_{1}(t)\right| \approx \Delta n(t)=n_{\max }(t)-n_{\min }(t),
$$

which is calculated as the difference between the maximum and minimum values of $n(x, t)$ at a particular time. Note that this is a more general method for calculating the refractive index modulation than used in [1] or [8], as it does not need the assumption that $\Delta n$ is linearly related to polymer concentration; indeed, for the early stages of recording, it can be seen that all species concentrations make a (nonlinear) contribution. 


\section{$3 \quad$ Numerical simulations}

The equations (4)-(6), together with the relevant initial and boundary conditions, were integrated numerically using a standard implicit finite difference scheme, for short $\left(t_{e}=10 \mathrm{~s}\right)$ and long $\left(t_{e}=100 \mathrm{~s}\right)$ exposure times. The numerical scheme was implemented in $\mathrm{C}$, while the data manipulation and graphics were performed using the IDL (Interactive Data Language) software. The total integration time was $t=100 \mathrm{~s}$ in all simulations (although the full range may not be shown in all diagrams).

We choose three values for the polymerization rate constant, $F_{0}=0.1 \mathrm{~s}^{-1}$, $F_{0}=1 \mathrm{~s}^{-1}$ and $F_{0}=10 \mathrm{~s}^{-1}$ (which correspond to regimes of low, moderate and high intensity recording), and for each of these values we study the dependence of the first harmonic amplitude $n_{1}(t)$ (or refractive index modulation), distortion from illumination pattern, $d(t)$, and refractive index profile, $n(x, t)$, on recording frequency $\Lambda$ and "immobilization" parameter $\gamma$. These numerical simulations are presented in subsections B and $\mathrm{C}$ below. The different curves shown in Figures 3-12 correspond to spatial frequencies between 100 and 2000 lines $/ \mathrm{mm}\left(\Lambda=5 \times 10^{-7}-10^{-5} \mathrm{~m}\right)$, although not all frequencies are shown in all diagrams in order to avoid cluttering.

Recall that the parameter $\gamma$ in this model represents the (non-dimensional) rate of conversion of mobile to immobile chains and depends on physical properties such as permeability of the photopolymer or amount of crosslinker present. Numerical values of $\gamma$ are difficult to determine experimentally so in our simulations we examine values related to those of polymerization rates, e.g. $\gamma=\beta / 10, \gamma=\beta$ or $\gamma=10 \beta$ (where $\beta=t_{0} F_{0}$ is the non-dimensional polymerization rate). Large values of $\gamma$ imply faster cross-linking and immobilization of the produced polymer chains and is also equivalent to creation of less permeable material. On the other hand, the permeability of the binder is one of the factors influencing the rate of immobilization of polymer chains since it determines how easily two shorter chains can move and interact to create a larger immobile polymer chain.

\subsection{The effect of binder on refractive index modulation}

Before starting the simulations on the influence of immobilization rate on final refractive index modulation and fidelity of the recorded pattern, studies on the influence of the binder refractive index were carried out. The time evolution of the refractive index modulation of the photopolymer system, as given by numerical simulations of (4)-(6) and (8), is sensitive to the values chosen for the refractive indices of the individual components. For 
example, if we vary the refractive index of the binder while keeping all other parameters constant, as given by (9), we obtain the curves in Figures 1-2. A low recording frequency $\left(\Lambda=10^{-5} \mathrm{~m}\right)$ was chosen for the simulation in Figure 1(a) and Figure 2(a) while a high frequency example $\left(\Lambda=10^{-6} \mathrm{~m}\right)$ is shown in Figure 1(b) and Figure 2(b). In all these simulations we used $F_{0}=0.1 \mathrm{~s}^{-1}$ and $\gamma=0.1$ while the exposure time is $t_{e}=10 \mathrm{~s}$.
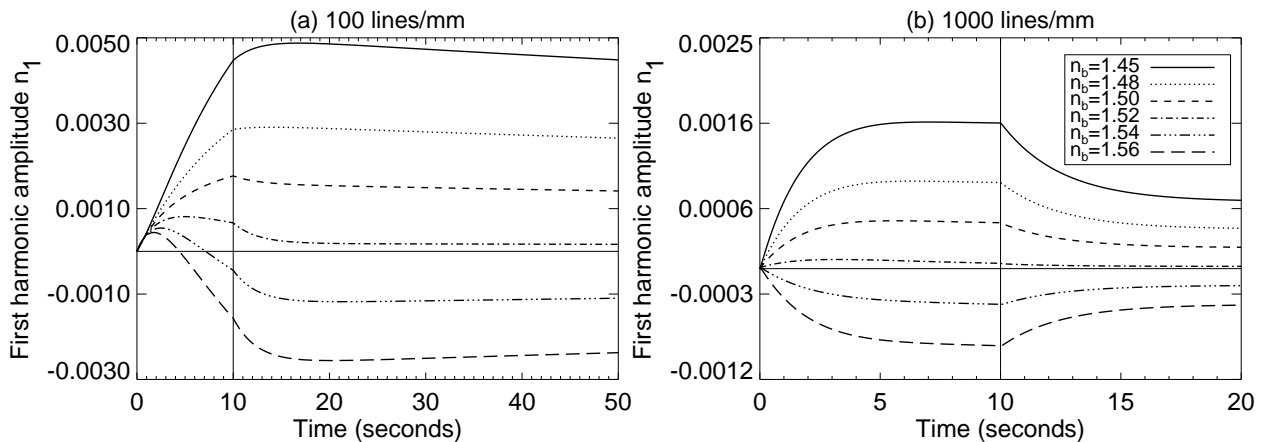

Figure 1: First harmonic amplitude for different values of binder refractive index, for (a) low recording frequency $\left(\Lambda=10^{-5} \mathrm{~m}\right)$ and (b) high frequency $\left(\Lambda=10^{-6} \mathrm{~m}\right)$. The legend applies to both (a) and (b). In both figures, $F_{0}=0.1 \mathrm{~s}^{-1}, \gamma=0.1$ and $t_{e}=10 \mathrm{~s}$.
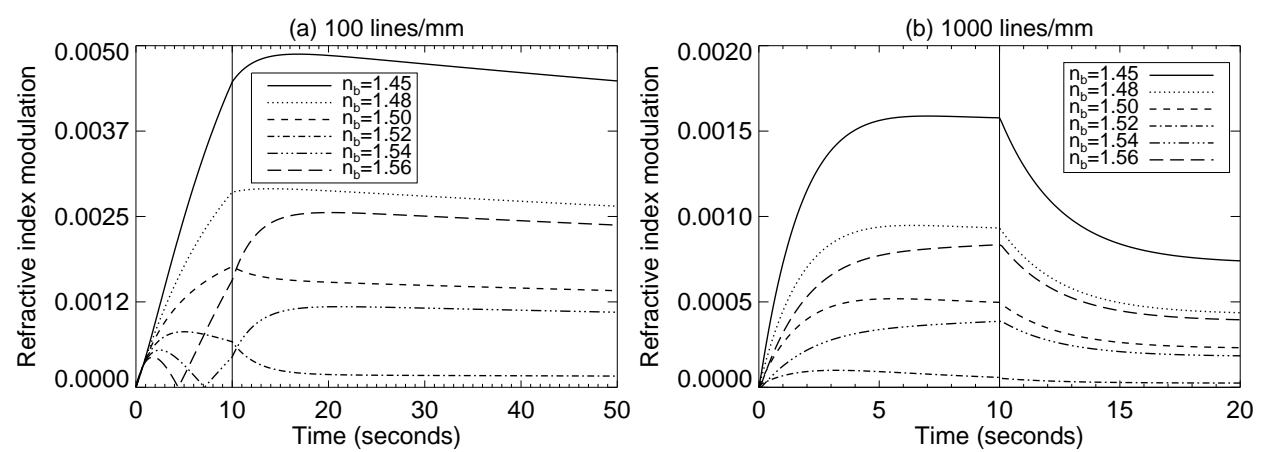

Figure 2: Refractive index modulation (as given by $\left|n_{1}(t)\right|$ ) for different values of binder refractive index, for (a) low recording frequency $\left(\Lambda=10^{-5} \mathrm{~m}\right)$ and (b) high frequency $\left(\Lambda=10^{-6} \mathrm{~m}\right)$. All values are the same as in Figure 1.

Figures 1(a) and (b) show the evolution of the first harmonic amplitude, $n_{1}(t)$, which decreases and becomes negative when the refractive index of 
the binder is higher than that of polymer, $n_{b}>n_{p}$, as the refractive index $n(x, t)$ is half a wavelength out of phase with the illumination pattern. By contrast, Figure 2 shows the absolute value of the first harmonic amplitude, $\left|n_{1}(t)\right|$, which is a better measure for the refractive index modulation. From this figure we notice that the long term value of the refractive index modulation depends on the difference between $n_{b}$ and $n_{p}$, regardless of which one is higher (for example, $n_{b}=1.48$ and $n_{b}=1.56$ produce similar long term behaviour in both (a) and (b), since $n_{p}=1.52$ ). Note that, as the remainder of this paper deals exclusively with the case $n_{b}<n_{p}$, the first harmonic amplitude will remain positive at all times and will be used as an approximation for refractive index modulation.

The simulations presented in Figures 1-2 reveal that a choice of a binder with lower refractive index is beneficial when a larger refractive index modulation is sought. Also, it can be seen from these graphs that, at very low spatial frequencies (for example 100 lines $/ \mathrm{mm}$ ), the refractive index modulation displays post-exposure growth, a fact which has been observed experimentally (see, for example, [12], [19]). The holographic recording typically leads to an increase of the density of the bright fringes due to photopolymerization and concentration driven monomer diffusion. In the case when the refractive index of the binder is lower than the refractive index of the polymer the refractive index modulation initially grows, since the difference between the refractive indices in the bright and the dark regions can only increase; however, after the short exposure time, the refractive index modulation decreases. The decrease is smaller at the lower spatial frequency since the distance over which the diffusion of mobile polymer chains takes place is one order of magnitude larger and thus the effect of the diffusion on smearing the refractive index profile is smaller. When $n_{b}>n_{p}$, the intervals of increase and decrease for the first harmonic amplitude are reversed, as seen in Figure 1.

\subsection{Low intensity recording}

The case of slow polymerization rate, $F_{0}=0.1 \mathrm{~s}^{-1}$, is shown in Figures 36 for two values of the immobilization parameter $\gamma$. Figure 3 shows the evolution of the first harmonic amplitude $n_{1}(t)$ for short and long exposures and low immobilization rate $\gamma=0.01$ while Figure 5 shows similar graphs for a higher immobilization constant, $\gamma=1$. The grating distortion $d(t)$, which was defined in (10), is plotted in Figures 4 and 6 , together with the refractive index spatial profile, $n(x, t)$, at the end of exposure $\left(t_{e}=100 \mathrm{~s}\right)$.

Figure 3 shows that the refractive index modulation decreases faster with 

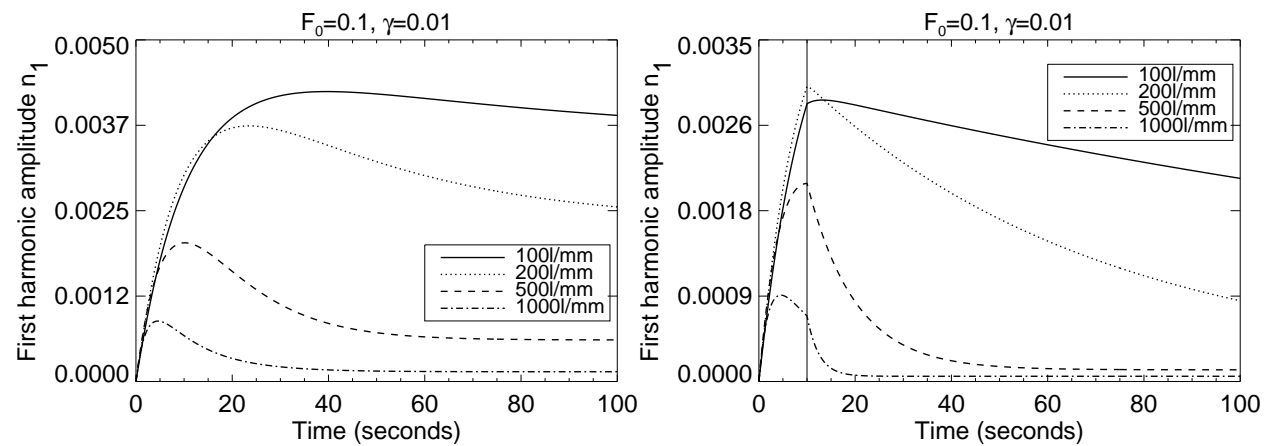

Figure 3: Low polymerization rate, $F_{0}=0.1$, and low immobilization constant, $\gamma=0.01$. The first harmonic amplitude $n_{1}(t)$ is shown for long exposure $t_{e}=100 \mathrm{~s}$ (left)and short exposure $t_{e}=10 \mathrm{~s}$ (right).
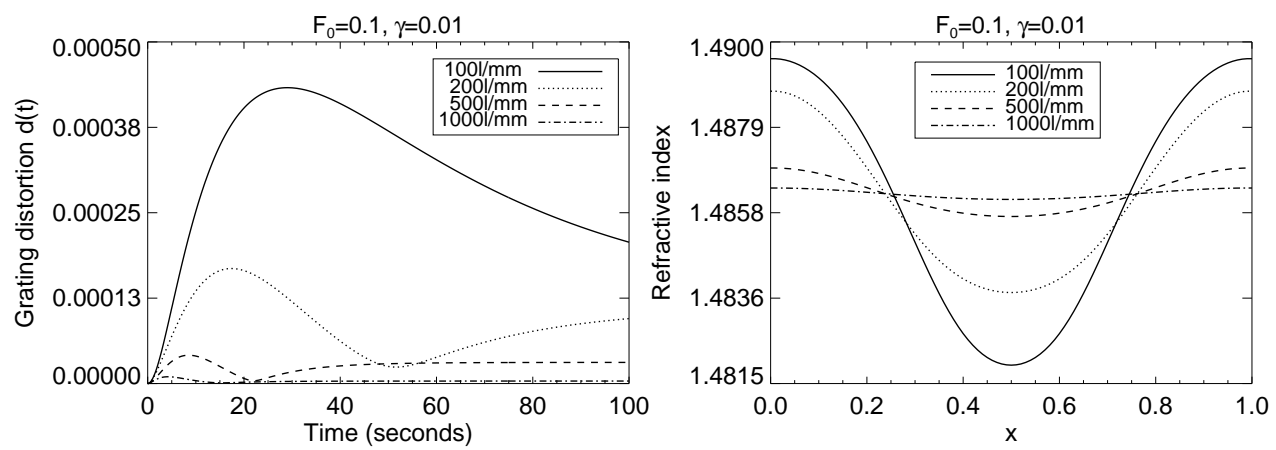

Figure 4: Low polymerization rate, $F_{0}=0.1$, and low immobilization constant, $\gamma=0.01$. The grating distortion, $d(t)$, is shown on the left and the spatial profile, $n(x, 100)$ on the right. The exposure time is $t_{e}=100$ s for both graphs. 
increasing recording frequency while Figure 5 shows that, for the same spatial frequency, increased immobilization rates lead to higher refractive index modulation values. Also, longer exposure times are more beneficial in the case of slow immobilization (Figure 3). In the case of faster immobilisation longer exposure time does not lead to significant improvement in the final refractive index modulation (Figure 5 ). Note that when $F_{0}=0.1$, the value of the nondimensional factor $K$ (the ratio of diffusion to polymerization rates, defined in (7)) varies from $K=0.1$ (achieved at a frequency of 100 lines $/ \mathrm{mm}$ ) to $K=40$ (at 2000 lines $/ \mathrm{mm}$ ). The results in Figure 3 show that the use of low intensity of recording is beneficial at low spatial frequencies and detrimental at high spatial frequencies, as long as the rate of immobilization is low. The picture changes significantly when the immobilization rate is two orders of magnitude faster (Figure 5) when steady growth reaching saturation is observed in long exposure simulations. The maximum refractive index modulation at this relatively low intensity of recording is observed at a spatial frequency of 200 lines $/ \mathrm{mm}\left(\Lambda=5 \times 10^{-6} \mathrm{~m}\right)$. The reason for the higher value observed at $200 \mathrm{l} / \mathrm{mm}$ than at $100 \mathrm{l} / \mathrm{mm}$ is the shorter distance travelled by the monomer molecules from dark to bright areas. This allows for a better mass transport and a larger density change to be achieved. The grating period and the distances that the molecules have to travel at 100 and 200 lines/mm are large and the detrimental effect of mobile terminated/unterminated polymer chains diffusion is negligible. In addition, due to the higher distortion at this spatial frequency (see Figure 6), the amount of light diffracted into higher orders will be higher at 100 lines/mm than at 200 lines $/ \mathrm{mm}$ and, for that reason, the first harmonic amplitude at 100 lines $/ \mathrm{mm}$ is slightly smaller at these conditions of recording.

Comparing the results presented in Figures 3-6, the following observations can be made: 1) the drop of diffraction efficiency after short exposure significantly decreases with increasing immobilization rate, as the polymer chains are prevented from moving and consequently smearing the created refractive index pattern; 2) at low intensity of recording, the best results for the refractive index modulation are achieved at low spatial frequency, which was also observed experimentally in [25]; 3) faster immobilization is more important for high rather than low spatial frequencies at this intensity; 4) very little distortion of the recorded refractive index profile is observed at this low intensity of recording. 

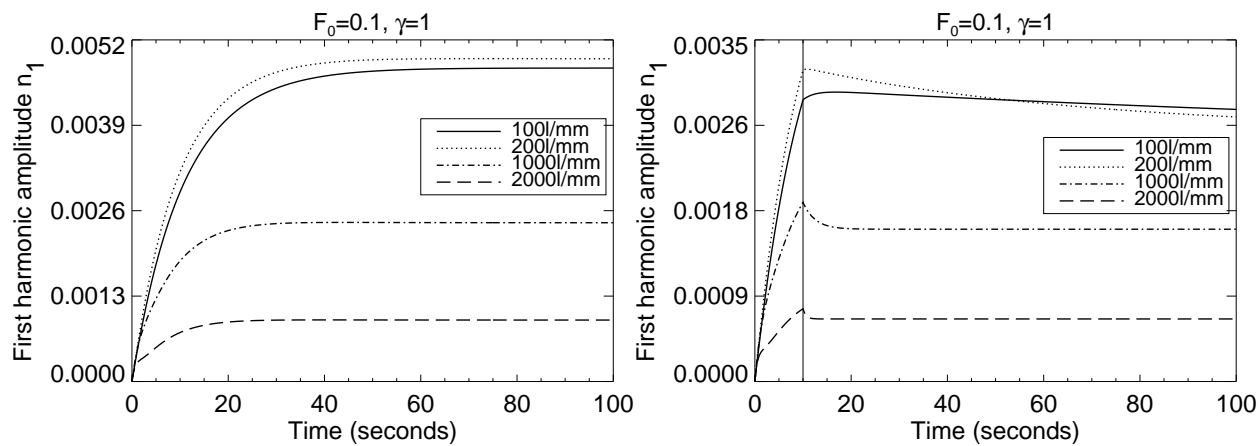

Figure 5: Low polymerization rate, $F_{0}=0.1$, and higher immobilization constant, $\gamma=1$. The first harmonic amplitude $n_{1}(t)$ is shown for long exposure $t_{e}=100 \mathrm{~s}$ (left) and short exposure $t_{e}=10 \mathrm{~s}$ (right).
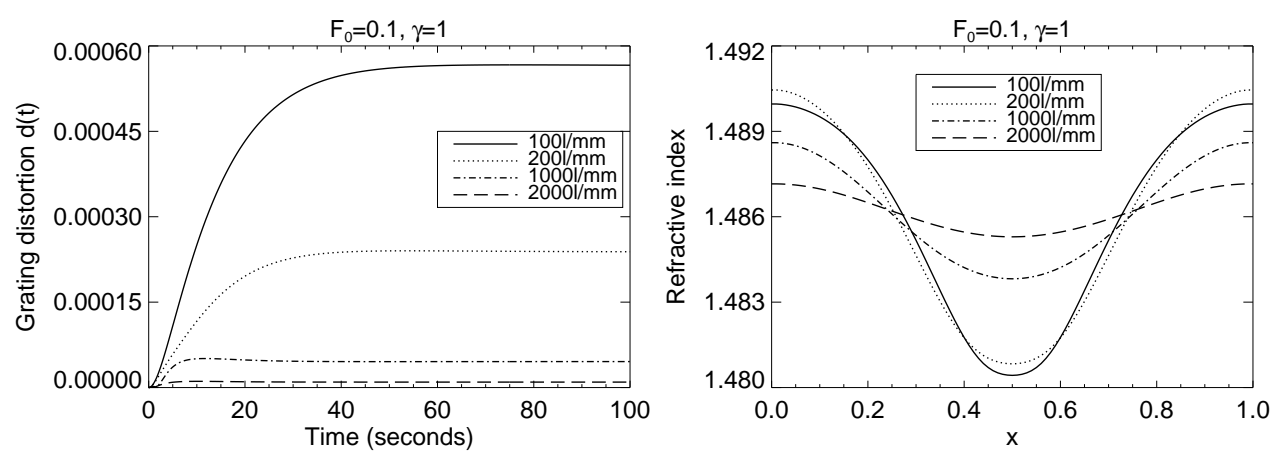

Figure 6: Low polymerization rate, $F_{0}=0.1$, and higher immobilization constant, $\gamma=1$. The grating distortion, $d(t)$, is shown on the left and the spatial profile, $n(x, 100)$ on the right. The exposure time is $t_{e}=100 \mathrm{~s}$ for both graphs. 


\subsection{Moderate and high intensity recording}

Figures $7-12$ show the cases of moderate $\left(F_{0}=1 \mathrm{~s}^{-1}\right)$ and high $\left(F_{0}=10 \mathrm{~s}^{-1}\right)$ polymerization rates. At these values, we found little difference between an exposure of $t_{e}=10 \mathrm{~s}$ and $t_{e}=100 \mathrm{~s}$ and therefore only the long exposure simulations were displayed. When $F_{0}=1$, the diffusion to polymerization ratio $K$ varies from $K=0.01$ (for 100 lines $/ \mathrm{mm}$ ) to $K=4$ (at 2000 lines $/ \mathrm{mm}$ ), while for $F_{0}=10$ we obtain the range $K=0.001-0.4$. We note from Figures 8-9 and 11-12 that, at small values of $K$ (corresponding to low recording frequencies), the refractive index variation exhibits distortions from the initial sinusoidal profile. This phenomenon will be further investigated in Section 4B.

It is observed that an increased rate of immobilization (which can be achieved, for example, by fast cross-linking) is beneficial for both medium and high intensity of recording. Contrary to the case of low recording intensity, medium and high intensities lead to better results and higher refractive index modulation at high spatial frequency. From Figures $7-10$ it is seen that the optimal frequency of recording and immobilization rate (the values which lead to the highest refractive index modulation and lowest distortion) depend on the spatial frequency. For example, moderate intensity $F_{0}=1$ and fast immobilization $\gamma=10$ are optimal for 500 lines $/ \mathrm{mm}$, while high intensity $F_{0}=10$ and fast immobilization $\gamma=10$ are best for the higher frequency 2000 lines $/ \mathrm{mm}$. The exact pattern for this dependence is difficult to establish as it results from a complex interplay between processes such as monomer and polymer diffusion as well as polymer immobilization, all of which having different effects in different parameter regimes.

\section{Perturbation analysis}

The behaviour of the solutions in two extreme cases, $K \ll 1$ and $K \gg 1$ (hence the diffusion rate is much smaller or much larger than the polymerization rate) can be studied analytically using perturbation methods, a standard tool in mathematical modelling (see, for example, [26]). Since $\varepsilon=D_{p} / D_{m}$ is a small parameter, we expand the concentration functions $m, p$ and $q$ in asymptotic series using powers of $\varepsilon$ thus reducing the system (4)-(6) to a sequence of simpler problems which can be solved sequentially. In particular, this approach will show the appearance of distortions for small $K$ values. As this analysis is concerned with the long term behaviour of the monomer and polymer concentrations under constant light exposure, we let $\Phi(t) \equiv 1$ in (4)-(6). 

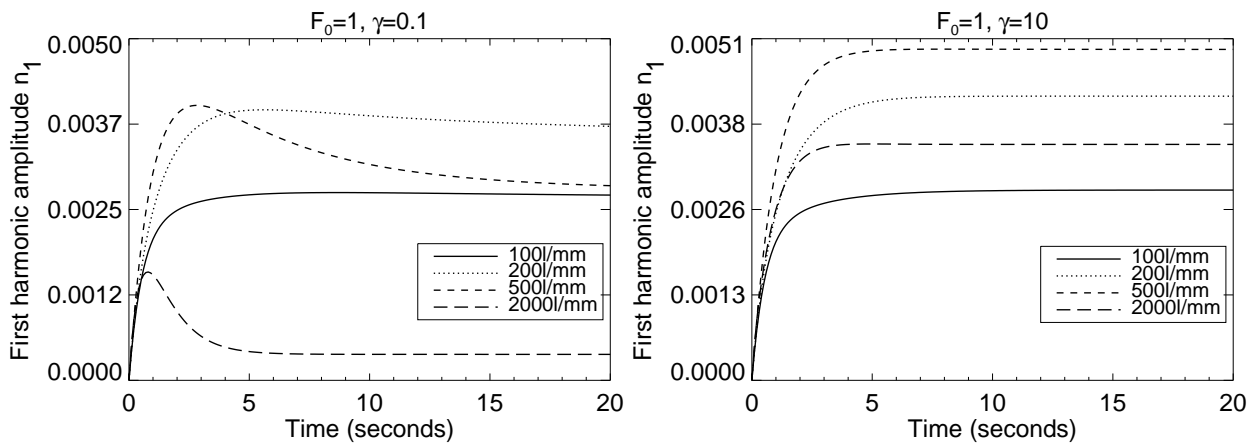

Figure 7: Moderate polymerization rate, $F_{0}=1$. The evolution of the first harmonic amplitude $n_{1}(t)$ is shown for low immobilization constant, $\gamma=0.1$ (left) and high immobilization constant, $\gamma=10$ (right). The exposure time is $t_{e}=100$ s for all curves.

\subsection{Polymerization rate less than diffusion rate $(K \gg 1)$}

Without loss of generality we choose $\alpha=D_{m} t_{0} / \Lambda^{2}=1$ (achieved, for example, with $t_{0}=1 s, \Lambda=10^{-6} \mathrm{~m}$ ) and $\beta=\sqrt{\varepsilon}$ (corresponding to $F_{0}=0.1 \mathrm{~s}^{-1}$ ), which gives $K=10$. We also assume the immobilization rate to be smaller than the polymerization rate, hence we take $\gamma=\varepsilon$. The subsequent analysis can be easily modified to deal with other parameter regimes which lead to large $K$ values. Figure 13 shows numerical simulations of the spatial profiles of monomer and polymer concentrations, $m, p$ and $q$, as well as refractive index, $n$, for various times between 0 and 100s (in these graphs, short and long polymers refer to mobile and immobile chains, respectively). Note that the mobile polymer concentration first increases due to polymerization but then decreases as the immobilization process starts to dominate.

Multiple timescales expansions (see [26]) are appropriate since the polymers evolve more slowly than the monomer and can be used to approximate the initial behaviour for all species. Using the short time $t$ and long time $\tau=\varepsilon^{1 / 2} t$, we write

$$
\begin{aligned}
m(x, t, \tau)= & m_{0}(x, t, \tau)+\sqrt{\varepsilon} m_{1}(x, t, \tau)+\varepsilon m_{2}(x, t, \tau)+ \\
& + \text { higher order terms (h.o.t), }
\end{aligned}
$$

with similar expressions for $p$ and $q$. Substituting this expansion into (4) 

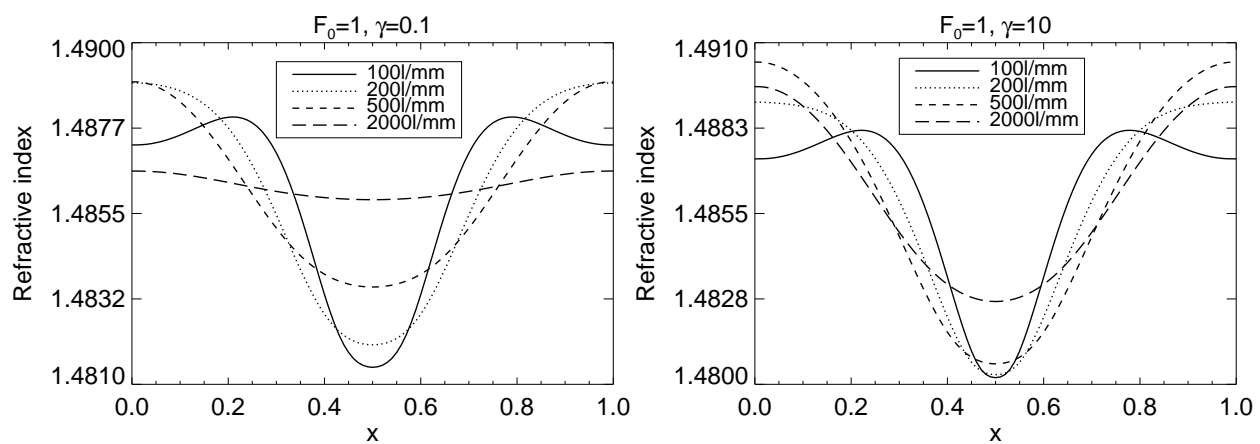

Figure 8: Moderate polymerization rate, $F_{0}=1$. The refractive index spatial profile $n(x, 100)$ is shown for low immobilization constant, $\gamma=0.1$ (left) and high immobilization constant, $\gamma=10$ (right). The exposure time is $t_{e}=100 \mathrm{~s}$ for all curves.

and equating coefficients of each power of $\varepsilon$, we obtain

$$
\begin{array}{cc}
\text { Order } 1: & \frac{\partial m_{0}}{\partial t}=\frac{\partial^{2} m_{0}}{\partial x^{2}}, \quad m_{0}(x, 0,0)=1 \\
\text { Order } \sqrt{\varepsilon}: & \frac{\partial m_{1}}{\partial t}=\frac{\partial^{2} m_{1}}{\partial x^{2}}-\left(\frac{\partial m_{0}}{\partial \tau}+f(x) m_{0}\right), \\
m_{1}(x, 0,0)=0,
\end{array}
$$

and similar problems at higher orders, all satisfying zero-flux boundary conditions. Solving these problems sequentially, we obtain

$$
\begin{aligned}
m(x, t, \tau)= & \mathrm{e}^{-\tau}+\sqrt{\varepsilon}\left[\frac{1}{8 \pi^{2}} \tau \mathrm{e}^{-\tau}+\right. \\
& \left.\frac{1}{4 \pi^{2}} \mathrm{e}^{-\tau}\left(\mathrm{e}^{-4 \pi^{2} t}-1\right) \cos (2 \pi x)\right]+ \text { h.o.t }
\end{aligned}
$$

and a similar approach for (5)-(6) gives asymptotic expansions for the polymers

$$
\begin{aligned}
& p(x, t, \tau)=1-\mathrm{e}^{-\tau}+\frac{\mathrm{e}^{-\tau}-\mathrm{e}^{-4 \pi^{2} \tau}}{4 \pi^{2}-1} \cos (2 \pi x)+\text { h.o.t } \\
& q(x, t, \tau)=\sqrt{\varepsilon} \int_{0}^{\tau} p_{0}^{2}(x, s) d s+\text { h.o.t. }
\end{aligned}
$$

These are valid up to $t=O(1 / \sqrt{\varepsilon}$ ) (although the monomer expansion can be shown to hold as $t \rightarrow \infty$ ) and on this time scale it can be seen that the 

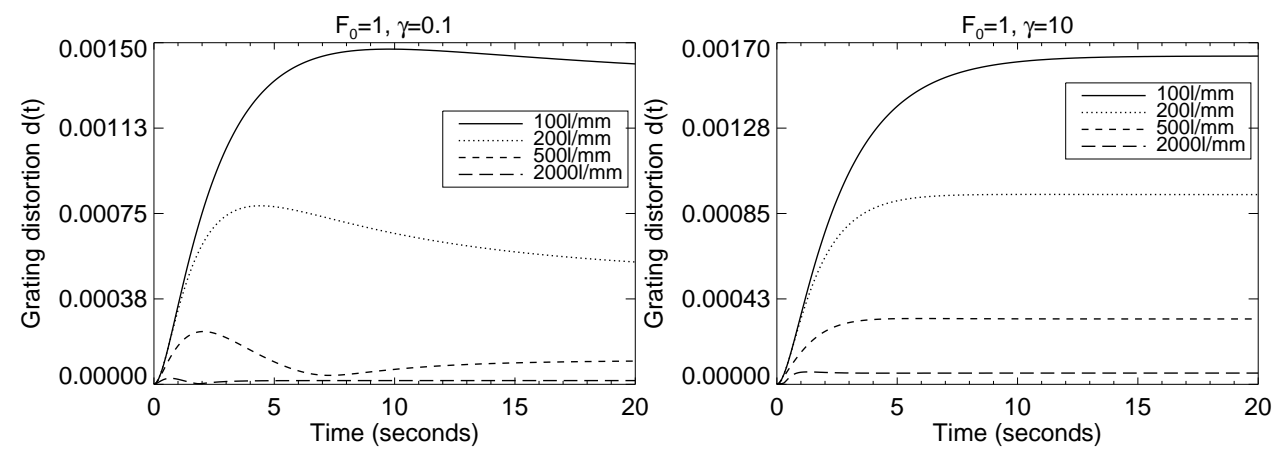

Figure 9: Moderate polymerization rate, $F_{0}=1$. The grating distortion $d(t)$ is shown for low immobilization constant, $\gamma=0.1$ (left) and high immobilization constant, $\gamma=10$ (right). The exposure time is $t_{e}=100 \mathrm{~s}$ for all curves.

leading order spatial dependence is sinusoidal, or constant (as in the case of monomer, which only depends on the slow time $\tau$ ). These asymptotic approximations give good agreement with the numerical solutions shown in Figure 13. The evolution of the refractive index modulation and grating distortion for this particular choice of physical constants $\left(F_{0}=0.1, \Lambda=\right.$ $\left.10^{-6}, \gamma=0.01\right)$ can be seen in Figures $3-4$. The distortion measure in this case is low, which confirms the sinusoidal profile calculated in this section.

It is evident from the asymptotic approximations and numerical simulations presented above that this regime is characterized by monomer and polymer profiles with little spatial variation, due to the relatively short diffusion distances, and consequently a fast decaying refractive index modulation. However, even under these conditions of high diffusion to polymerization rate, the refractive index contrast can be improved if the polymer immobilization rate is increased (for example, by using a more efficient cross-linker), as shown in Figure 5.

\subsection{Diffusion rate less than polymerization rate $(K \ll 1)$}

To illustrate the case when the diffusion rate is much slower than the polymerization rate we choose, for example, $\beta=t_{0} F_{0}=1, \alpha=\gamma=\varepsilon \ll 1$, which gives $K=0.01$. (We take $\Lambda=10^{-5} \mathrm{~m}$ and $F_{0}=1 \mathrm{~s}^{-1}$.) A standard 

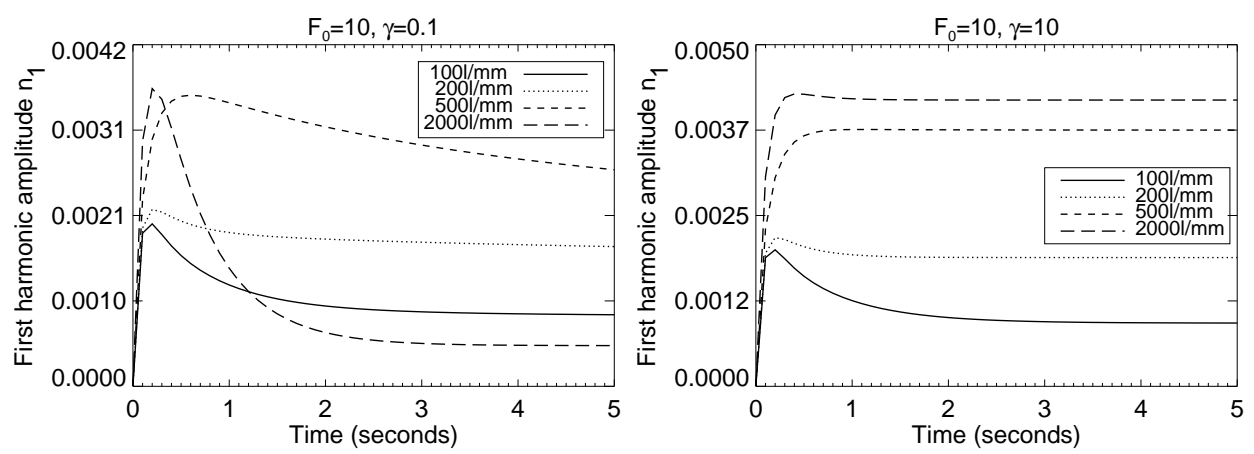

Figure 10: High polymerization rate, $F_{0}=10$. The evolution of the first harmonic amplitude $n_{1}(t)$ is shown for low immobilization constant, $\gamma=0.1$ (left) and high immobilization constant, $\gamma=10$ (right). The exposure time is $t_{e}=100$ s for all curves.

perturbation approach applied to (4) gives

$$
\begin{aligned}
m(x, t)=\mathrm{e}^{-(1+\cos (2 \pi x)) t}+\varepsilon \frac{2}{3} \pi^{2} t^{2} \mathrm{e}^{-(1+\cos (2 \pi x)) t} \times \\
\times(t+3 \cos (2 \pi x)-t \cos (4 \pi x))+\text { h.o.t },
\end{aligned}
$$

which is valid for $t \ll 1 / \sqrt{\varepsilon}$; however this expansion fails for large $t$ and $x \approx \frac{1}{2}$ (the point of zero illumination) due to the presence of secular terms. This suggests the existence of an internal layer, centred at $x=\frac{1}{2}$, where the solution changes very fast and can be analysed using the change of variables $\xi=\left(x-\frac{1}{2}\right) \varepsilon^{-1 / 4}, \tau=\varepsilon^{1 / 2} t$. The non-dimensional monomer equation (4) becomes

$$
\frac{\partial m}{\partial \tau}=\frac{\partial^{2} m}{\partial \xi^{2}}-m\left(2 \pi^{2} \xi^{2}-\varepsilon^{\frac{1}{2}} \frac{2 \pi^{4}}{3} \xi^{4}+\cdots\right)
$$

where $-\infty \leq \xi \leq \infty, \tau \geq 0$ and we used a Taylor approximation for the illumination function,

$$
f(x)=1+\cos (2 \pi x)=1-\cos \left(2 \pi \xi \varepsilon^{\frac{1}{4}}\right)=2 \pi^{2} \xi^{2} \varepsilon^{\frac{1}{2}}+\cdots
$$

The asymptotic expansion

$$
m(\xi, \tau)=m_{0}(\xi, \tau)+\varepsilon m_{1}(\xi, \tau)+\cdots
$$

substituted into (12) gives the following equation for the leading order monomer approximation

$$
\frac{\partial m_{0}}{\partial \tau}=\frac{\partial^{2} m_{0}}{\partial \xi^{2}}-2 \pi^{2} \xi^{2} m_{0}, \quad-\infty \leq \xi \leq \infty, t \geq 0
$$



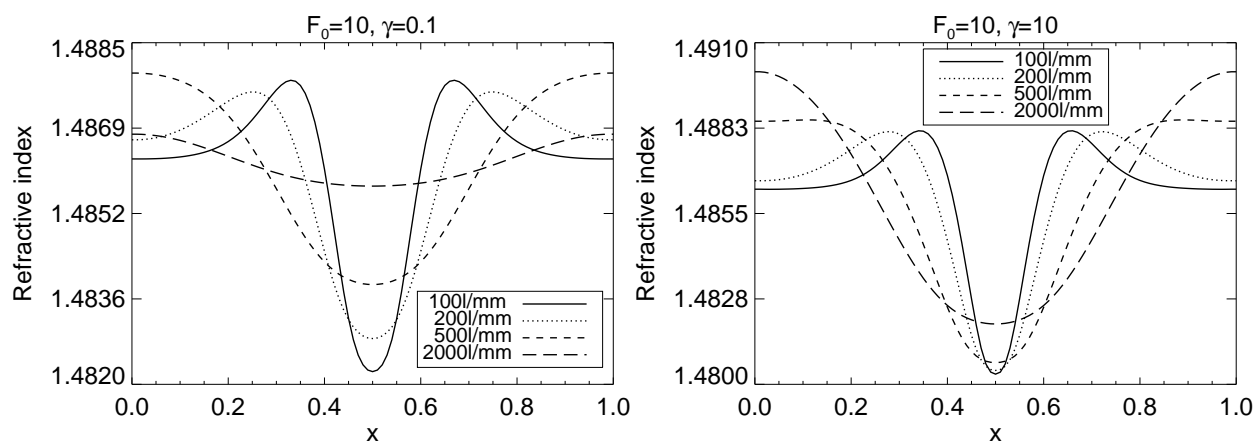

Figure 11: High polymerization rate, $F_{0}=10$. The refractive index spatial profile $n(x, 100)$ is shown for low immobilization constant, $\gamma=0.1$ (left) and high immobilization constant, $\gamma=10$ (right). The exposure time is $t_{e}=100 \mathrm{~s}$ for all curves.

where $\frac{\partial m_{0}}{\partial \xi}( \pm \infty)=0$. Using the separation of variables assumption $m_{0}(\xi, \tau)=$ $A(\xi) B(\tau)$ we obtain the problems

$$
\begin{aligned}
& \frac{d B}{d \tau}=\lambda B, \\
& \frac{d^{2} A}{d \xi^{2}}-\left(\lambda+2 \pi^{2} \xi^{2}\right) A=0, \quad \frac{d A}{d \xi}( \pm \infty)=0,
\end{aligned}
$$

where $\lambda$ is the separation constant. The problem in (15) is a non-dimensional form of the quantum harmonic oscillator (or the Schrödinger equation with quadratic potential) and its solutions are well known. It can be shown (see, for example, [27]) that the only possible eigenvalues are

$$
\lambda_{n}=-\pi \sqrt{2}(2 n+1), \quad n=0,1,2 \ldots
$$

with corresponding eigenfunctions

$$
A_{n}(\xi)=\mathrm{e}^{-\frac{\pi}{\sqrt{2}} \xi^{2}} H_{n}\left(\left(2 \pi^{2}\right)^{1 / 4} \xi\right)
$$

where $H_{n}$ are the Hermite polynomials. The first problem, (14), then yields $B(\tau)=\mathrm{e}^{-\lambda_{n} \tau}$ and hence the general solution for the first order problem (13) is given by

$$
m_{0}(\xi, \tau)=\sum_{n=0}^{\infty} C_{2 n} \mathrm{e}^{-\pi \sqrt{2}(4 n+1) \tau-\frac{\pi}{\sqrt{2}} \xi^{2}} H_{2 n}\left(\left(2 \pi^{2}\right)^{1 / 4} \xi\right)
$$



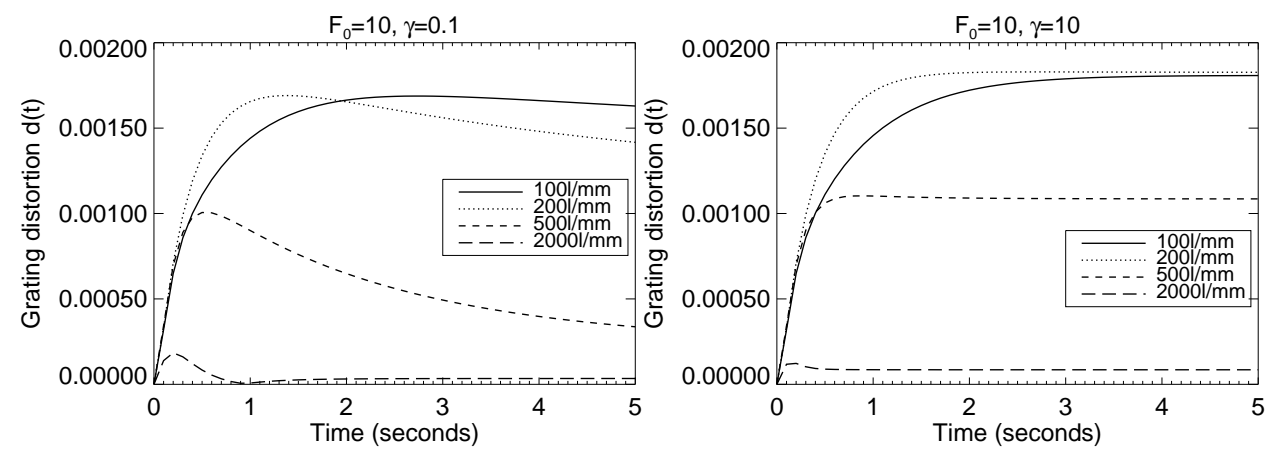

Figure 12: High polymerization rate, $F_{0}=10$. The grating distortion $d(t)$ is shown for low immobilization constant, $\gamma=0.1$ (left) and high immobilization constant, $\gamma=10$ (right). The exposure time is $t_{e}=100 \mathrm{~s}$ for all curves.

where we have only retained the even eigenfunctions. The constants $C_{2 n}$ are determined using the initial condition $m_{0}(\xi, 0)=1$ together with properties of the Hermite polynomials:

$$
C_{2 n}=\frac{\sqrt{2}}{2^{n}(2 n) ! !} .
$$

The first terms in the expansion shown in (16) are therefore

$$
\begin{gathered}
m_{0}(\xi, \tau)=\sqrt{2} \mathrm{e}^{-\pi \sqrt{2} \tau-\frac{\pi}{\sqrt{2}} \xi^{2}}+\mathrm{e}^{-5 \pi \sqrt{2} \tau-\frac{\pi}{\sqrt{2}} \xi^{2}}\left(2 \pi^{2} \xi^{2}-\frac{1}{\sqrt{2}}\right) \\
+\frac{1}{4 \sqrt{2}} \mathrm{e}^{-9 \pi \sqrt{2} \tau-\frac{\pi}{\sqrt{2}} \xi^{2}}\left(8 \pi^{2} \xi^{4}-12 \sqrt{2} \pi \xi^{2}+3\right)+\cdots
\end{gathered}
$$

It is easily seen that for fixed $\tau(\tau=O(1))$, the amplitudes decay very fast as $n \rightarrow \infty$ and that the second and subsequent terms make a very small contribution to $m_{0}$.

The inner and outer expansions (17) and (11) (which represent solution approximations inside and outside the "dark" layer) accurately describe the behaviour of the monomer concentration for all times and can be used to determine similar approximations for the polymer functions; all these approximations match the numerical solutions shown in Figure 14. In this case the refractive index profile no longer resembles the sinusoidal illumination as it develops multiple maxima per grating period. These distortions typically appear on the longer time scale of $\tau$ and could therefore be avoided by using shorter exposure times. The two peaks in the polymer and refractive 

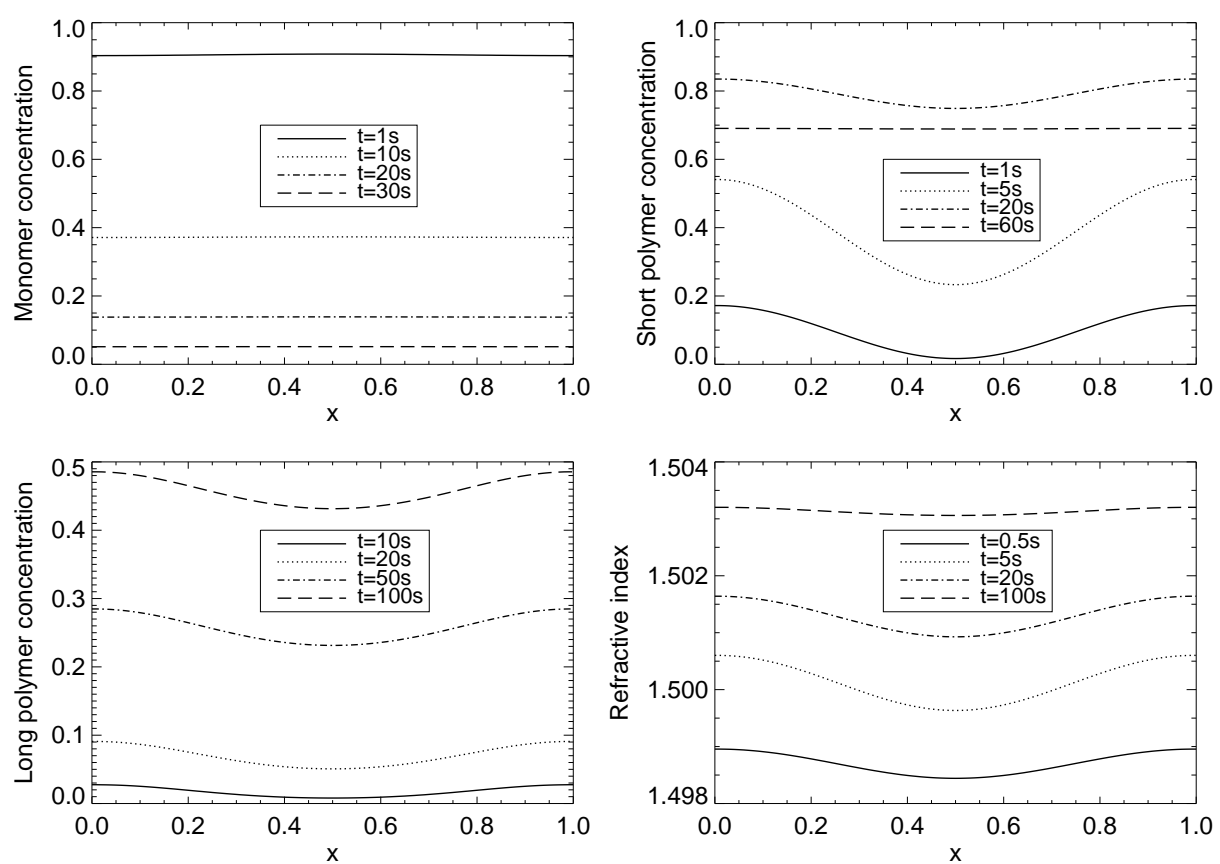

Figure 13: Time evolution of monomer, polymers and refractive index profiles for $K \gg 1$. Here, $\alpha=1, \beta=\sqrt{\varepsilon}=0.1, \gamma=0.01$ and $K=10$. Exposure time is $t_{e}=100 \mathrm{~s}$. 
index profiles, adjacent to the dark region, have already been reported in the literature (see, for example, [11], [8]).

The evolution of the refractive index modulation and grating distortion for this particular choice of physical constants $\left(F_{0}=10, \Lambda=10^{-5}, \gamma=0.1\right)$ can be seen in Figures 10, 12. In this case only a modest improvement is observed in the refractive index modulation when the immobilization rate is increased by a factor of 100 . The distortion function $d(t)$ is high, which implies that higher harmonic amplitudes make a significant contribution to the refractive index in this case. The analytical approach presented in this section, seems to suggest that, in the $K \ll 1$ regime, the component concentrations might be more accurately described by different eigenfunction expansions, rather than the standard Fourier cosine series.
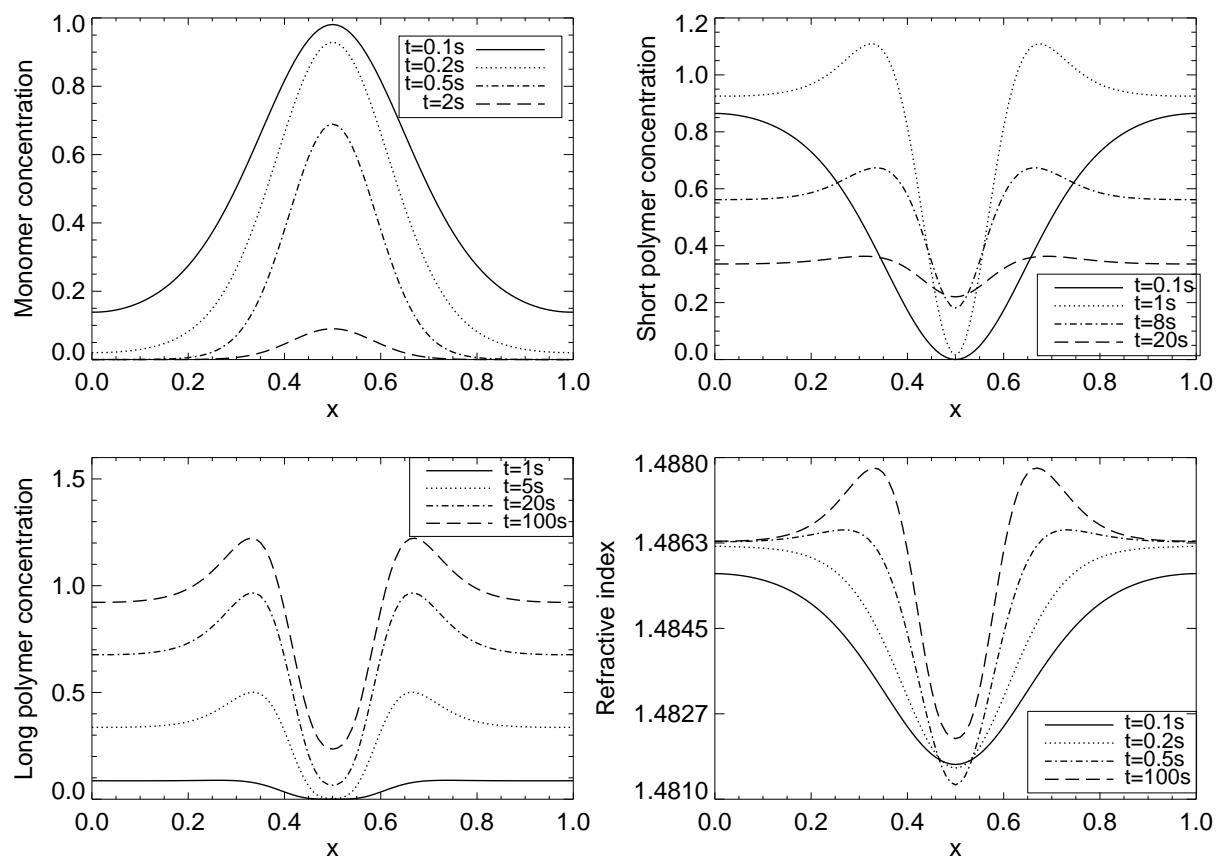

Figure 14: Time evolution of monomer, polymers and refractive index profiles for $K \ll 1$. Here, $\alpha=\varepsilon=0.01, \beta=1, \gamma=0.1$ and $K=0.01$. Exposure time is $t_{e}=100 \mathrm{~s}$. 


\section{Conclusions}

In this paper we presented a mathematical model for holographic grating formation in an acrylamide-based photopolymer system. The equations are derived from standard physical laws (diffusion, mass conservation, photopolymerization) and no a priori assumptions were made regarding the solution behaviour. The model assumes short polymer chains are free to diffuse and introduces an immobilization rate which is determined by chain growth and cross-linking. Numerical simulations were carried out in order to assess the effect of recording parameters such as illumination frequency and intensity, as well as material permeability, on refractive index modulation, refractive index profile and grating distortion.

The behaviour of the photopolymer system was seen to be qualitatively different in two extreme regimes, characterized by polymerization rate much larger than diffusion rate $(K \gg 1)$ and polymerization rate much slower than diffusion rate $(K \ll 1)$. A perturbation analysis, verified by numerical integration of the model equations, shows that the monomer and polymer concentrations, as well as the refractive index, have a weakly sinusoidal spatial variance when $K \gg 1$ but display large distortions due to higher harmonics when $K \ll 1$. (Note that the model derived in [19] assumes that the monomer concentration decreases homogeneously due to high monomer mobility; however, our analysis shows that this assumption, while approximately correct when $K \gg 1$, would not be appropriate when $K \ll 1$.)

The model presented here suggests that, for achieving good response at high spatial frequency of recording, it is beneficial to work with high intensities and fast immobilization rates. By contrast, good response at low spatial frequency of recording requires lower recording intensities, [25]. Fast immobilization is desirable but not as critical as in the case of high spatial frequency. It is also observed from numerical simulations that, for any polymerization rate $F_{0}$ and immobilization parameter $\gamma$, the grating distortion is always highest at the lowest recording frequency $\Lambda$.

It was demonstrated that the model can predict the diffraction gratings dynamics at long exposure times and the obtained values for the refractive index modulation are comparable to those reported in the literature.

\section{Acknowledgments}

The authors wish to thank Prof. Vincent Toal (Industrial and Engineering Optics Centre, Dublin Institute of Technology) for useful discussions and 
advice.

\section{References}

[1] G. Zhao and P. Mouroulis, "Diffusion model of hologram formation in dry photopolymer materials", J. Mod. Opt. 41, 1929-1939 (1994).

[2] S. Piazzola and B. Jenkins, "First-harmonic diffusion model for holographic grating formation in photopolymers", J. Opt. Soc. Am. B 17, 1147-1157 (2000).

[3] V. Moreau, Y. Renotte and Y. Lion, "Characterization of DuPont photopolymer: determination of kinetic parameters in a diffusion model", Appl. Opt. 41, 3427-3435 (2002).

[4] V.L. Colvin, R.G. Larson, A.L. Harris and M.L. Schilling, "Quantitative model of volume hologram formation in photopolymers", J. Appl. Phys. 81, 5913-5923, (1997).

[5] S. Blaya, L. Carretero, R. Mallavia, A. Fimia and R.F. Madrigal, "Holography as a technique for the study of photopolymerization kinetics in dry polymeric films with a nonlinear response", Appl. Opt. 38, 955-962 (1999).

[6] J.H. Kwon, H.C. Hwang and K.C. Woo, "Analysis of temporal behavior of beams diffracted by volume gratings formed in photopolymers", J. Opt. Soc. Am. B 16, 1651-1657 (1999).

[7] C. Neipp, S. Gallego, M. Ortuno, A. Marquez, M.L. Alvarez, A. Belendez and I. Pascual, "First-harmonic diffusion-based model applied to a polyvinyl-alcohol acrylamide-based photopolymer", J. Opt. Soc. Am. B 20, 2052-2060, (2003).

[8] J.T. Sheridan and J.R. Lawrence, "Nonlocal-response diffusion model of holographic recording in photopolymer", J. Opt. Soc. Am. A 17, 11081114 (2000).

[9] J.T. Sheridan, M. Downey and F.T. O'Neill, "Diffusion based model of holographic grating formation in photopolymers: Generalised non-local material responses", J. Opt. A: Pure and Appl. Opt. 3, 477-488 (2001). 
[10] J. Lawrence, F. O'Neill and J.T. Sheridan, "Adjusted intensity nonlocal diffusion model of photopolymer grating formation", J. Opt. Soc. Am. B 19, 621-629 (2002).

[11] S.D. Wu and E.N. Glitsis, "Holographic grating formation in photopolymers: analysis and experimental results based on a nonlocal diffusion model and rigorous coupled-wave analysis", J. Opt. Soc. Am. B 20, 1177-1188, (2003).

[12] I. Naydenova, R. Jallapuram, R. Howard, S. Martin, and V. Toal, "Investigation of the Diffusion Processes in a Self-Processing AcrylamideBased Photopolymer System", Appl. Opt. 43, 2900-2905 (2004).

[13] Y. Shiga and C. Egami, "Shift-multiplexed microhologram fabrication with photoisomeric chromophores", Appl. Opt. 51, 2847-2855 (2012).

[14] G. Zhao and P. Mouroulis, "Second order grating formation in dry holographic photopolymers", Opt. Comm. 115, 528-532 (1995).

[15] S. Liu, M.R. Gleeson, J. Guo and J.T. Sheridan, "High Intensity Response of Photopolymer Materials for Holographic Grating Formation", Macromolecules 43, 22, 9462-9472, (2010).

[16] M.R. Gleeson, D. Sabol, S. Liu, C.E. Close, J.V. Kelly and J.T. Sheridan, "Improvement of the spatial frequency response of photopolymer materials by modifying polymer chain length", J. Opt. Soc. Am. B 25, 396-406 (2008).

[17] S. Martin, I. Naydenova, R. Jallapuram, R. Howard and V. Toal, "Twoway diffusion model for the recording mechanism in a self developing dry acrylamide photopolymer", Proc. SPIE 6252, 62525-625217 (2006).

[18] T. Babeva, I. Naydenova, D. Mackey, S. Martin and V. Toal, "Twoway diffusion model for short exposure holographic grating, formation in acrylamide based photopolymers", J. Opt. Soc. Am. B, 27, No. 2, (2010).

[19] M. Květoň, P. Fiala and A. Havránek, "Polymer Holography in Acrylamide-Based Recording Material", Holography, Research and Technologies, Prof. Joseph Rosen (Ed.), ISBN: 978-953-307-227-2, InTech, DOI: $10.5772 / 14564,(2011)$. 
[20] C. Meka, R. Jallapuram, I. Naydenova, S. Martin and V. Toal, "Development of a panchromatic acrylamide-based photopolymer for multicolor reflection holography", Appl. Optics, 49 (8), 1400-1405, (2010).

[21] I. Naydenova, R. Jallapuram, V.Toal and S. Martin, "A visual indication of environmental humidity using a colour changing hologram recorded in a self-developing photopolymer", Appl. Phys. Lett., 92, 031109 (2008).

[22] I. Naydenova, R. Jallapuram, V. Toal and S. Martin, "Characterisation of the humidity and temperature responses of a reflection hologram recorded in acrylamide-based photopolymer", Sensors and Actuators B: Chemical, 139 (1), 35-38, (2009).

[23] B.A. Kowalski, A.C. Urness, M.E. Baylor, M.C. Cole, W.L. Wilson and R.R. McLeod, "Quantitative modeling of the reaction/diffusion kinetics of two-chemistry diffusive photopolymers", Opt. Mat. Express, 4 (8), 1669, DOI:10.1364/OME.4.001668, (2014).

[24] D. Mackey, T. Babeva, I. Naydenova and V. Toal, "A diffusion model for spatially dependent photopolymerization", in Progress in Industrial Mathematics at ECMI 2008 (Mathematics in Industry), A.D. Fitt, J. Norbury, H. Ockendon and E. Wilson, eds. (Springer, 2010), pp. 253259.

[25] H. Akbari, I. Naydenova and S. Martin, "Using acrylamide based photopolymers for fabrication of holographic optical elements in solar energy applications", Appl. Opt. 53, 1343-1353 (2014).

[26] J.K. Kevorkian and J.D. Cole, Multiple Scale and Singular Perturbation Methods (Springer Applied Mathematical Sciences Series, 1996).

[27] D.J. Griffiths, Introduction to Quantum Mechanics (Pearson Prentice Hall, 2004). 